\title{
Simultaneous two-phase flow measurements in a high-speed particle-laden under-expanded jet
}

\author{
Miguel X. Diaz-Lopez ${ }^{1}$, Juan Sebastian Rubio ${ }^{1}$, and Rui Ni ${ }^{1}$ \\ ${ }^{1}$ Johns Hopkins University, Baltimore, MD 21218
}

\begin{abstract}
The objective of this study is to understand the dynamics of a high-speed particle-laden under-expanded jet, motivated by landings on extraterrestrial bodies. In this setup, inertial particles are entrained and accelerated by an under-expanded jet. But, due to their inertia, the particle velocity is significantly lower than that of the surrounding gas close to the nozzle, so the two phases are coupled through aerodynamic drag. Sub-micron oil droplets are dispensed upstream to serve as tracers, whose velocity is determined through a PIV system; inertial particles, after image segmenting is performed to separate them from PIV data, will be tracked over time with a PTV system. This was accomplished with a single laser pulse and the camera straddle time to produce image pairs and shorten the pulse width. The results will help to understand particle-laden flow in a new regime where the background flow is compressible and the Mach number based on the slip velocity is not negligible, which may help to pave a foundation for future studies in compressible multiphase flows.
\end{abstract}

\section{Introduction}

Particle-laden compressible flows are ubiquitous in nature and in many engineering applications, including volcano eruptions Cigala et al. (2017), particle ingestion in gas turbines Dunn (2012), and powered descent and landing on extraterrestrial bodies Morris et al. (2015). Despite its importance, experimental work in this area is scarce, especially in the compressible regime, where two phases are strongly coupled through aerodynamic drag. One of the key challenges is to simultaneously track inertial particles and measure the surrounding gas flows with velocities at $O(10-1000 \mathrm{~m} / \mathrm{s})$ Buchmann et al. (2014).

Previous studies of particle dynamics in a compressible shear layer showed that when the particle Stokes number becomes large, the slip Mach number can be supersonic, resulting in local bow shocks around particles Samimy and Lele (1991). When the particle mass loading increases, the effect could be so strong that the Mach disk location can be moved upstream close to the nozzle exit due to the momentum transfer between the two phases Sommerfeld (1994). However, these observations were limited to qualitative measurements without access to the dynamics of both phases at the same time.

The goal of this work is to simultaneously measure the gas and particle velocities using particle image velocimetry (PIV) and particle tracking velocimetry (PTV), which has been conducted before in incompressible flows. Previous simultaneous PIV/PTV measurements have used dual-camera systems with optical discrimination Elhimer et al. (2016) and single-camera systems with image segmentation of raw images Kiger and Pan (2000) to obtain both particle and velocity fields. Using our ultra-high-speed diagnostics systems, we plan to extend the simultaneous PIV/PTV technique to the compressible flow regime.

\section{Experimental Methodology}

The Johns Hopkins University Jet (JHU Jet) facility is a cold-air system that accelerates gas to subsonic, sonic, and supersonic speeds. To study the dynamics of inertial particles, a particle injector and accelerator were employed to inject particles from a particle chamber into the gas stream along the centerline of the jet. Additional technical details of the facility can be found in Ref. Kim et al. (2020).

An aerosolizer is used to suspend tracer particles, consisting of DEHS mineral oil droplets with a modal diameter of $0.25 \mu \mathrm{m}$. The sufficient large scale separation between inertial particles with size in the range of $O(10)-O(100) \mu \mathrm{m}$ and aerosol tracer particles makes it easy to segment their images from the PIV data. 
In many PIV applications, the pulse width of the laser is infinitesimally small because of its short span compared to the rest of the time scales. However, since one of the goals of this experiment is to obtain slip velocities close to the inertial particles, a small field of view (FOV) is necessary to sufficiently resolve both phases within this regime. The FOV is 13.5 by $8 \mathrm{~mm}$ with particles traveling at $340 \mathrm{~m} / \mathrm{s}$ (sonic speed) or even greater. For this reason, the particle will move several pixels within the 200 ns pulse width of the laser causing traces in the image. The solution to this issue was to utilize a single pulse which is placed within the straddle time of the camera. With the straddle time being slightly shorter than the laser pulse width, the laser is cut such that the rising edge of the pulse is in the first frame while the falling edge of the laser is in the second frame. This technique creates image pairs for PIV analysis while also cutting the pulse width for each frame. One issue that arose from this is that the decay time for the pulse is much slower than the rise time causing there to still be small traces in the second image but significantly reduced from previous experiments. More work will have to be done to remove traces from both phases. Finally, the acquired images were segmented to separate the two phases then analyzed using PIV and PTV methods, respectively.

\section{Results}

After analyzing the data, there were slight overestimates for both the inertial and tracer particles velocities when compared to simulation. This is likely due to traces in the second image making it difficult for the PIV algorithm to distinguish where the particle is exactly. Even with this overestimation, the overall trend matches literature and simulation closely. Further work will have to be done to either fix the traces in post processing or modify the method to remove the traces. Figure 1 shows sample images (left) with their respective segmentation and the normalized velocities of both the inertial and tracer particles (right).
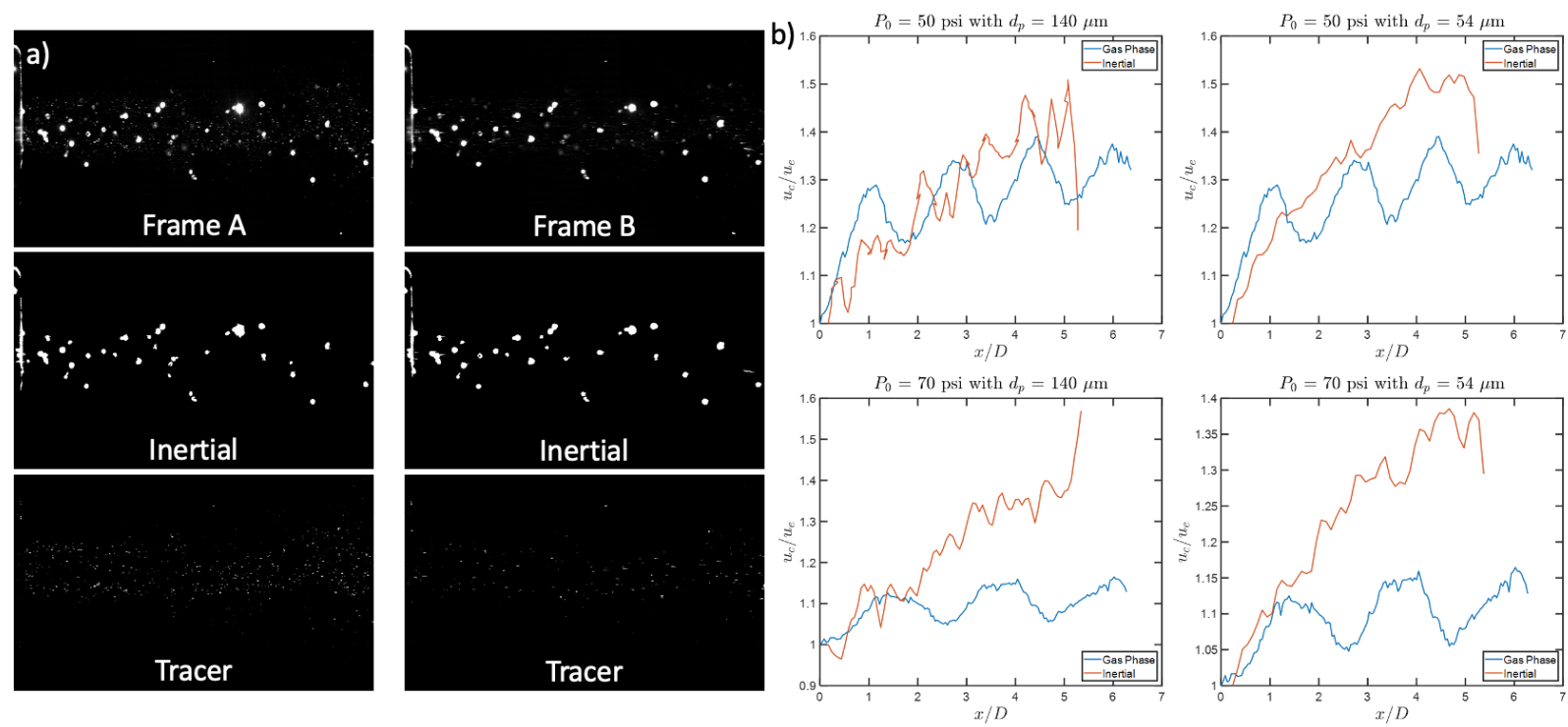

Figure 1: a) Sample PIV/PTV correlated frames at the top with segmented images to separate inertial from tracer particles underneath. b) Normalized results between inertial phase (orange) and tracer particles (blue). Both are normalized with their respective exit velocities at the nozzle.

\section{Conclusion}

A system consisting of a compressible particle-laden jet facility and a PIV/PTV diagnostic method was constructed to understand the interaction between inertial particles with the surrounding compressible gas. Although this diagnostic method has been used before in other types of incompressible multiphase flow, it would be the first attempt in the compressible regime. Valuable lessons and new results will be shared with the community to advance the diagnostic methods and our understanding of the compressible multiphase flow. 


\section{Acknowledgements}

This material is based upon work supported by the National Aeronautics and Space Administration under Grant No. 80NSSC19K0488 issued through Plume Surface Interaction (PSI) by the Space Technology Mission Directorate Game Changing Development. We would also like to thank Kyle D. Gilroy, Ph.D. and Andy Kubit from Vision Research, AMETEK, Materials Analysis Division for playing essential roles in helping to design the synchronization setup.

\section{References}

Buchmann NA, Cierpka C, Kähler CJ, and Soria J (2014) Ultra-high-speed 3D astigmatic particle tracking velocimetry: application to particle-laden supersonic impinging jets. Experiments in Fluids 55:1842

Cigala V, Kueppers U, Peña Fernández JJ, Taddeucci J, Sesterhenn J, and Dingwell DB (2017) The dynamics of volcanic jets: Temporal evolution of particles exit velocity from shock-tube experiments. Journal of Geophysical Research: Solid Earth 122:6031-6045

Dunn MG (2012) Operation of Gas Turbine Engines in an Environment Contaminated With Volcanic Ash. Journal of Turbomachinery 134. 051001

Elhimer M, Praud O, Marchal M, Cazin S, and Bazile R (2016) Simultaneous piv/ptv velocimetry technique in a turbulent particle-laden flow. Journal of Visualization 20

Kiger KT and Pan C (2000) PIV Technique for the Simultaneous Measurement of Dilute Two-Phase Flows. Technical report

Kim T, Ni R, Capecelatro J, Yao Y, Shallcross GS, Mehta M, and Rabinovitch J (2020) The dynamics of inertial particles in underexpanded jets: An experimental study. in AIAA Scitech 2020 Forum. page 1326

Morris A, Goldstein D, Varghese P, and Trafton L (2015) Approach for modeling rocket plume impingement and dust dispersal on the moon. Journal of Spacecraft and Rockets 52:1-13

Samimy M and Lele SK (1991) Motion of particles with inertia in a compressible free shear layer. Physics of Fluids A: Fluid Dynamics 3:1915-1923

Sommerfeld M (1994) The structure of particle-laden, underexpanded free jets. Shock Waves 3:299-311 